\title{
Thermodynamic Analysis of the Reverse
}

\author{
Joule-Brayton Cycle Heat Pump
}

for Domestic Heating

\author{
A.J. White \\ Hopkinson Laboratory, \\ Cambridge University Engineering Department, \\ Trumpington Street, Cambridge, CB2 1PZ, UK. \\ Email:ajw36@cam.ac.uk
}

\begin{abstract}
The paper presents an analysis of the effects of irreversibility on the performance of a reverse Joule-Brayton cycle heat pump for domestic heating applications. Both the simple and recuperated (regenerative) cycle are considered at a variety of operating conditions corresponding to traditional (radiator) heating systems and lowtemperature under-floor heating. For conditions representative of typical central heating in the UK, the simple cycle has a low work ratio and so very high compression and expansion efficiencies and low pressure losses are required to obtain a worthwhile COP. An approximate analysis suggests that these low loss levels would not necessarily be impossible to achieve, but further investigation is required, particularly regarding irreversible heat transfer to and from cylinder walls. In principle, recuperation improves the cycle work ratio, thereby making it less susceptible to losses, but in practice this advantage is compromised when realistic values of recu-
\end{abstract}


perator effectiveness are considered.

Key words: Heat Pump, Reverse Joule-Brayton, Domestic Heating.

\section{INTRODUCTION}

Current concerns over energy resources and $\mathrm{CO}_{2}$ emissions have led to renewed interest in the use of heat pumps for domestic heating. Traditionally, ground-source devices have been favoured for this purpose since stable ground temperatures offer the potential for a higher coefficient of performance (COP) compared with air-source heat pumps. However, relatively few residential properties have sufficient land space to install the extensive underground heat exchange loop required for ground source devices and so their uptake has been limited. (Borehole ground loops can overcome this problem, but installation costs are still very high.) Despite the theoretically lower values of COP, air-source devices may therefore have greater potential for exploitation. Cockroft and Kelly [1], for example, conclude that air-source heat pumps offer the greatest potential to reduce $\mathrm{CO}_{2}$ emissions amongst a variety of competing technologies for domestic heating and power.

The present paper explores the thermodynamic performance of an open aircycle heat pump, based on the reverse Joule-Brayton cycle (henceforth referred to as the RJB cycle). This cycle was first proposed for heating applications in 1852 by Kelvin, who referred to his device as a 'heat multiplier' [2]. The equivalent refrigeration cycle (which is known variously as the Brayton refrigeration cycle, the reverse Brayton cycle and the gas refrigeration cycle), also dates 
back to the nineteenth century when it was employed as a means of cooling on board cargo ships [3]. More recently, heat pumps based on both the simple [4] and regenerative [5] forms of the RJB cycle have been proposed, whilst Braun et al. [6] have analysed an air cycle heat pump for drying applications. The RJB cycle also finds application in aircraft cooling and air liquefaction, either as an open or a closed cycle device, and a number of studies have been published in this context. Of particular note is the work by Chen, Wu and co-workers [7-14] in which so-called finite-time thermodynamics is applied to analyse and optimise simple and regnerative RJB cycles operating in conjunction with fixed and variable temperature thermal reservoirs. Other cycle analyses have been undertaken in the context of some novel applications including cryogenic refrigeration (e.g., $[15,16])$ and lunar base cooling [17]. None of the above publications, however, addresses directly the issues relevant to a domestic heating application, which is the focus of the present work.

Sisto [18] undertook cycle calculations for a turbomachinery-based RJB cycle aimed at heating applications and concluded that a COP of about 2 could be achieved. However, even state-of-the-art turbomachines cannot achieve compression and expansion efficiencies that are required to provide a competitive COP. The use of reciprocating devices is therefore assumed in the present study and the method of treating losses is accordingly modified. A prototype "Brayton" domestic water heating system based on reciprocating devices was in fact built and tested in the 1970s [19], but achieved a COP of just 1.26. This poor performance would appear to stem from high pressure losses through valves and from stray heat losses. These issues are also addressed in the present 
work.

As a heating device, the open-loop reverse Joule-Brayton cycle has a number of attractive features when compared to traditional vapour compression devices. Since the working fluid is ambient air, there are no refrigerant leakage problems, and there is no requirement for a low-temperature heat exchanger. (By contrast, a ground source heat pump usually employs two levels of heat exchange on the low temperature side.) The cycle is therefore very simple and, in its ideal (i.e., reversible) form, its COP matches that of a reverse Carnot cycle operating between temperature limits of the ambient air temperature and the radiator supply water temperature. Against these potential advantages, air-cycle devices have a tendency to be bulky due to the low density and thermal capacity of air, and at conditions typical of central heating systems the cycle has a very low work ratio, making it particularly susceptible to irreversibilities.

The objectives of the present study are to investigate the performance of both a simple and a recuperated (or "regenerative") RJB cycle and to determine the levels of thermodynamic loss that can be tolerated if a worthwhile COP is to be obtained for heating applications (i.e., above $\sim 3.5$, excluding electrical and mechanical losses). Some initial consideration is also given to the optimum operating conditions, but overall system optimisation (which requires details of the radiator "load line" and integration of performance over a typical heating season) has not been undertaken. The analysis presented is based on simple cycle calculations, assuming the working fluid (air) to behave as a perfect gas. This is a reasonable assumption with the possible exception of 
within the expansion process where moisture or ice precipitation may occur. Thermodynamic losses within the cycle are quantified by polytropic expansion and compression efficiencies and by pressure loss factors, estimates of which are obtained by considering an outline design for the heat pump.

\section{CYCLE DESCRIPTION AND OPERATING POINT}

Figure 1 shows a simplified layout of a reverse Joule-Brayton heat pump. Ambient air is drawn into the compressor at 1 and compressed to state 2, typically at about twice atmospheric pressure. The hot compressed air then enters a heat exchanger where it is cooled to state 3 , thereby delivering heat to the radiator circulating water (and / or the hot water system). Finally, the air is expanded through the reciprocating expander to atmospheric pressure and exits to the atmosphere at state 4 . The throttles $\mathrm{T} 1$ - T4 represent pressure losses in the compressor and expander inlet and exit valves, and in the heat exchanger and pipework.

For an electrically driven heat pump the overall COP is defined as the ratio between the thermal output and the electrical work input. Clearly this will be affected by mechanical (frictional) losses and electrical (motor and switching) losses, but since the focus here is upon thermodynamic behaviour, the COP will be computed on the basis of an ideal mechanical work input. The overall COP will be some 10 to $20 \%$ below this value. 


\subsection{Specification of Operating Point}

It is well-known that the maximum COP that can be achieved by a heat pump depends on the ratio between the source and sink temperatures. In the current context, the relevant temperatures are the outside air temperature, $T_{1}$, and the compressor delivery temperature, $T_{2}$, the latter being a few degrees above the radiator (or hot water) supply temperature. Except in the limiting case of the reversible cycle, the COP also depends on the temperature after the heat exchanger, $T_{3}$, which is dictated by the water temperature drop that can be achieved in the radiator system. In dimensionless terms, the operating point of the cycle is thus defined by the two parameters:

$$
\tau=\frac{T_{2}}{T_{1}} \quad \text { and } \quad \theta=\frac{T_{3}}{T_{1}}
$$

There is some scope for varying these parameters independently, but in practice they are linked by the "load line" of the heating system.

For the purposes of estimating pressure losses and providing an initial indication of performance, it is convenient to specify a reference operating point and this is taken as $\tau_{*}=1.216$ and $\theta_{*}=1.180$. This corresponds to the conditions listed in table 1 and is approximately representative of what might be achieved with a typical central-heating radiator system operating at reduced thermal output, and with a constant air-water temperature difference of $5{ }^{\circ} \mathrm{C}$ in the heat exchanger. (Note that the geometry described in table 1 is based on two compression and two expansion cyclinders, each pair being coupled on a single shaft, as in fig. 1.) 


\section{PERFORMANCE OF THE IDEAL CYCLE}

Figure 2 shows ideal and real reverse Joule-Brayton cycles plotted on a T-s diagram. For either cycle, provided all components (except the heat exchanger) are adiabatic, the $\mathrm{COP}$ is given by,

$$
\mathrm{COP}=\frac{q}{w_{c}-w_{e}}=\frac{c_{p}\left(T_{2}-T_{3}\right)}{c_{p}\left(T_{2}-T_{1}\right)-c_{p}\left(T_{3}-T_{4}\right)}
$$

The maximum COP will be achieved if all processes are also reversible (dashed line in the figure), in which case the temperature ratios across the compressor $\left(\tau_{c}=T_{2} / T_{1}\right)$ and expander $\left(\tau_{e}=T_{3} / T_{4}\right)$ are the same and equal to $\tau$. Eq.(1) then simplifies to:

$$
\mathrm{COP}_{\mathrm{i}}=\left(1-\frac{1}{\tau}\right)^{-1}
$$

This, as expected, is simply the reciprocal of the efficiency for an ideal gas turbine cycle, and it is notable that the expression is independent of the heat exchanger temperature drop, and hence of the thermal output, $q=c_{p}\left(T_{2}-T_{3}\right)$. For the reference operating conditions (table 1), the maximum COP is about 5.6 and requires a pressure ratio of roughly $2: 1$.

\section{DEPARTURES FROM IDEAL BEHAVIOUR}

Before embarking on a detailed analysis of how the various losses affect the $\mathrm{COP}$, it is worth making a few general comments. The net work input per $\mathrm{kg}$ of air for the ideal cycle is equal to the area enclosed by the p-v diagram, as shown in fig. 3. The striking feature of this diagram is that the enclosed area is very slender - i.e., the cycle has a very low work ratio (defined as the ratio 
between the net work input and the compression work input). This means that small fractional changes in either $w_{c}$ or $w_{e}$ have a large impact on the net work input and hence on the COP. To put this into perspective, at the reference operating point (at which $w_{c}$ and $q$ will be fixed), a $1 \%$ reduction in the expansion work results in the COP falling by almost $25 \%$. As shown in

fig. 3 , the work ratio is improved by increasing the temperature drop in the heat exchanger such that, unlike the ideal cycle, the COP of the real device is quite strongly dependent on $\theta$.

\subsection{Quantification of Losses}

The principal departures from ideal thermodynamic behaviour are caused by:

(i) Non-adiabatic compression and expansion.

(ii) Pressure losses in valves, pipework and the heat exchanger.

(iii) Irreversibility during compression and expansion.

Some of these effects are shown on the T-s diagram of fig. 2 (solid line), and the associated loss factors are defined below. Note that, since valve pressure losses are treated separately here, the main source of irreversibility during compression and expansion is thermal dissipation, as described in appendix B.

\section{Polytropic Compression and Expansion}

Compression and expansion irreversibilities are traditionally quantified by isentropic efficiencies. This is the approach adopted in, for example, $[9,12,17]$, 
and is suitable for adiabatic compression and expansion with turbomachinery. For reciprocating devices, it is advantageous to separate out the pressure losses associated with the various valves. Furthermore, isentropic efficiencies are not properly defined if the processes concerned involve heat transfer. For example, it is quite possible during a compression process for the effects of heat loss and irreversibility to cancel each other, giving a process that is isentropic but in no sense ideal. A means of simultaneously accounting for both heat losses (or gains) and irreversibility is therefore required.

In the present analysis, compression heat losses are quantified by a fractional heat loss factor, $\alpha_{c}$, defined by:

$$
d q_{c}=\alpha_{c} d w_{c}
$$

where $d q_{c}$ is the heat lost and $d w_{c}$ is the work input (both per unit mass of air) during an infinitesimal compression process. The reversible work input for such a process is equal to $d p / \rho$, irrespective of whether or not the process is adiabatic. Irreversibility may therefore be quantified by writing:

$$
d w_{c}=\frac{d p}{\eta_{c} \rho}
$$

where $\eta_{c}$ is the compression (polytropic) efficiency.

Combining equations (3) and (4) and integrating (assuming $\alpha_{c}$ and $\eta_{c}$ remain constant) gives a polytropic relationship of the form,

$$
\tau_{c}=\beta_{c}^{\phi_{c}}
$$

where $\tau_{c}$ and $\beta_{c}$ are the compression temperature and pressure ratios respec- 
tively, and $\phi_{c}$ is a polytropic exponent, given by:

$$
\phi_{c}=\frac{\gamma-1}{\gamma}\left(\frac{1-\alpha_{c}}{\eta_{c}}\right)
$$

The compression work input is then found by applying the steady flow energy equation in conjunction with eq.(3), giving:

$$
w_{c}=\frac{c_{p} T_{1}\left(\tau_{c}-1\right)}{1-\alpha_{c}}
$$

Equivalent expressions to eq.(5) and eq.(7) may be derived for the expansion process, but in this case:

$$
\phi_{e}=\frac{\gamma-1}{\gamma}\left(1-\alpha_{e}\right) \eta_{e}
$$

and $\alpha_{e}$ must be interpreted as a fractional heat gain factor.

\section{Pressure Losses}

Pressure losses in the valves, pipework and heat exchanger all contribute to the expander seeing a lower pressure ratio than the compressor. These losses are represented here by fractional pressure loss factors, $f_{i}-$ e.g., for the compressor inlet valve,

$$
P_{1 a}=\left(1-f_{1}\right) P_{1}
$$

Similar expressions apply for other components, and the overall effect can be represented by a global pressure loss factor, $F$, defined by,

$$
\beta_{e}=\beta_{c} \prod_{i=1}^{4}\left(1-f_{i}\right)=\beta_{c}(1-F),
$$

To a good approximation, $F$ is given by summing the individual values of $f_{i}$, provided these are small. 


\subsection{Quantitative Effect of Losses on the COP}

By straightforward application of the steady flow energy equation to each component and incorporating eq.(7) and a similar expression for $w_{e}$, the COP for the irreversible cycle may be written as

$$
\mathrm{COP}=\frac{q}{w_{c}-w_{e}}=\frac{\tau-\theta}{\left(\frac{\tau-1}{1-\alpha_{c}}\right)-\theta\left(\frac{1-1 / \tau_{e}}{1-\alpha_{e}}\right)}
$$

(Note that the cycle temperature ratio, $\tau$ is equal to the compressor temperature ratio $\tau_{c}$ for the simple, unrecuperated cycle.) Combining eq.(10) with eq.(5) and an equivalent expression for the expander, gives the following relation between $\tau_{e}$ and $\tau_{c}$ :

$$
\tau_{e}=(1-F)^{\phi_{e}} \tau_{c}^{\eta_{c} \eta_{e}\left(1-\alpha_{e}\right) /\left(1-\alpha_{c}\right)} .
$$

This shows how pressure losses and polytropic efficiencies of less than unity result in the expander temperature ratio falling below that of the compressor, whereas heat losses and heat gains only have a significant effect if $\alpha_{c}$ and $\alpha_{e}$ differ.

\section{The Effect of Heat Losses}

For a reversible cycle in which the fractional heat losses and heat gains in the compressor and expander are balanced (i.e., $\alpha_{c}=\alpha_{e}=\alpha$ ), eq.(12) shows that the compression and expansion temperature ratios remain equal and so eq.(11) reduces to:

$$
\mathrm{COP}=(1-\alpha) \mathrm{COP}_{\mathrm{i}}
$$


Thus each $1 \%$ of heat loss in the compressor (and $1 \%$ heat gain in the expander) causes a $1 \%$ reduction in COP. However, if $\alpha_{c}$ and $\alpha_{e}$ differ, then changes to the COP may be much greater. Figure 4 shows T-s diagrams for reversible cycles with (a) no heat loss, (b) $\alpha_{c}=\alpha_{e}=0.05$ (i.e., $5 \%$ heat loss in the compressor and $5 \%$ heat gain in the expander) and (c) $\alpha_{c}=0.05$ but $\alpha_{e}=-0.05$ (i.e., $5 \%$ heat loss in both compressor and expander. Since these cycles are reversible, the areas enclosed by the T-s diagrams are equal to the net work input, whilst the heat delivered in each case is the same. It is clear from the figure that the reduction in COP will be very significant for case (c). It is also clear that the average compression and expansion temperatures are both likely to be above $T_{1}$ so that there is a tendency for heat loss from both devices (i.e., $\alpha_{c}>0$ but $\alpha_{e}<e$ ). It is therefore important to fully insulate the compressor and the expander, and henceforth it is assumed that sufficient insulation is used to make both devices adiabatic.

\section{The Effect of Irreversibilities}

Figure 5 shows the cycle COP plotted as a function of overall pressure loss factor, $F$, at the reference operating conditions, and for different values of compression and expansion efficiency. Bearing in mind that electrical and mechanical losses will cause a further reduction in performance, it is clear that only very small losses can be tolerated if a worthwhile COP is to be achieved at these conditions. For example, to obtain a thermodynamic COP of 3.5 would require compression and expansion efficiencies of $99.5 \%$ and pressure losses of less than $1 \%$, or some similar combination. Rudimentary estimates of $F$ and $\eta$ are presented in appendices $\mathrm{A}$ and $\mathrm{B}$ respectively, based on the outline 
geometry given in table 1 . Although subject to considerable uncertainty, these estimates suggest that such low levels of loss would be difficult to achieve at the specified values of $\theta$ and $\tau$. However, less stringent limits apply if $\theta$ can be reduced and if the operating point is optimised, as described in the following section.

\section{DESIGN POINT SELECTION}

The key to improving the cycle performance is to increase the cycle work ratio by increasing the air temperature drop in the heat exchanger, as described in section 4 . Ideally this would be achieved by reducing the radiator water return temperature, and hence reducing $\theta$, but whether or not this is possible depends on the type of radiator system; radiators designed for traditional central heating systems would probably have insufficient surface area to allow a significant reduction in $\theta$ and simultaneously provide the required thermal output. However, even at fixed $\theta$, improvements in performance may still be possible by increasing $\tau$, either by increasing the radiator water supply temperature, or by allowing the air and water temperatures to diverge in the heat exchanger. The purpose of this section is therefore to explore cycle optimisation on this basis. The results presented here are intended only to give an approximate indication of trends since the variation of $F, \eta_{c}$ and $\eta_{e}$ with operating conditions is not known with any certainty. In particular, no dependence of $\eta_{c}$ and $\eta_{e}$ on operating conditions is included in the calculations. 


\subsection{Optimisation at Fixed $\theta$}

For a specified thermal output, increasing the temperature drop in the heat exchanger leads to a lower air mass flow rate. Increasing $\tau$ at fixed $\theta$ therefore has a twofold effect: the cycle work ratio is improved, and pressure losses are also reduced due to the lower air velocities. It is assumed here that pressure losses scale approximately as the square of the mass flow rate and hence inversely with the square of the heat exchanger temperature drop. Thus,

$$
\frac{F}{F_{*}} \approx\left(\frac{\tau_{*}-\theta_{*}}{\tau-\theta}\right)^{2},
$$

where the asterisk denotes the reference conditions. Based on the estimates in appendix A, a value of $2.6 \%$ is used for $F_{*}$, except where otherwise stated.

Figure 6 shows the variation of $\mathrm{COP}$ with $\tau$ for $\theta=\theta_{*}=1.18$, corresponding to a radiator water return temperature of about $50{ }^{\circ} \mathrm{C}$. Curves are plotted for polytropic efficiencies of 98,99 and $100 \%$, and for $F_{*}=1.25,2.5$ and $5 \%$. These results demonstrate that the maximum COP is very sensitive to polytropic efficiency, but much less dependent on $F_{*}$. This is because as $F_{*}$ increases, the optimum operating point shifts to a higher value of $\tau$ (and hence a lower air mass flow rate) at which pressure losses are smaller. It should be borne in mind, however, that polytropic efficiencies may well decrease with decreasing air mass flow (since there is then more time for heat transfer), thereby counteracting this trend. Nonetheless, by judicious selection of the operating point, lower values of polytropic efficiency can be tolerated than initially suggested by fig. 5 . 


\subsection{Contours of COP in the $\tau-\theta$ Plane}

By use of underfloor heating or larger area radiators, it is possible to substantially reduce $\theta$ below the reference value of 1.18 , and it is therefore of interest to explore how the COP varies with both $\tau$ and $\theta$. Figure 7 shows contours of COP in the $\tau-\theta$ plane, for $F_{*}=2.6 \%$ and $\eta_{c}=\eta_{e}=99 \%$. With an underfloor heating system, values of $\theta$ as low as 1.12 should be possible (corresponding to $T_{3}=38{ }^{\circ} \mathrm{C}$ at an outside air temperature of $5{ }^{\circ} \mathrm{C}$ ), and the optimum value of $\tau$ is then 1.21 (corresponding to $T_{2}=63{ }^{\circ} \mathrm{C}$ for the same outside air temperature). This would give a thermodynamic COP of 4.5 based on the assumed loss parameters, as shown by the open circle plotted in the figure. Overall system optimisation would however require superimposing the radiator "load-line" on fig. 7 .

It is worth noting that if the heat pump is used to provide domestic hot water, then careful design of the hot water tank would permit $T_{3}$ to be reduced yet further. For at least part of the water heating process, values of $T_{3}$ of $\sim 20{ }^{\circ} \mathrm{C}$ should be possible, giving $\theta=1.054$ and a maximum COP of about 6 , assuming the same loss parameters.

\section{THE RECUPERATED CYCLE}

An alternative approach to improving the cycle work ratio is to "recuperate" heat from the air prior to expansion and transfer it to the compressor intake air, as shown in the T-s diagram of fig. 8. This may be achieved either by a direct (air-to-air) heat exchanger, or by first cooling the radiator return water 
with the inlet air and then exploiting this cooler water to obtain a lower air temperature prior to expansion. The latter method has the advantage that air pathways are less complex but requires two levels of heat exchange and thus results in a lower overall heat exchange effectiveness, $\epsilon$. For the ideal recuperated cycle (no losses, $\epsilon=1$ ) the COP is the same as for the simple reverse Joule-Brayton cycle (eq. 1), but the sensitivity to losses is much less: a $1 \%$ reduction in $w_{e}$ at the reference operating point results in only a $5 \%$ reduction in COP; one fifth of that for the simple cycle.

For the real cycle, with losses and $\epsilon<1$, eq.(12) relating $\tau_{e}$ and $\tau_{c}$ still applies, but $\tau_{c}$ and $\tau$ are no longer the same since part of the temperature rise ( $T_{1}$ to $T_{1 b}$ ) occurs by recuperation. Assuming an overall (air-to-air) heat exchange effectiveness of $\epsilon$ :

$$
T_{1 b}-T_{1}=T_{3}-T_{3 b}=\epsilon\left(T_{3}-T_{1}\right)
$$

The compressor temperature ratio is therefore given by:

$$
\tau_{c}=\frac{T_{2}}{T_{1 b}}=\frac{\tau}{1-\epsilon+\epsilon \theta}
$$

By reference to fig. 8, the COP of the recuperated cycle is simply:

$$
\mathrm{COP}=\frac{q}{w_{c}-w_{e}}=\frac{c_{p}\left(T_{2}-T_{3}\right)}{c_{p}\left(T_{2}-T_{1 b}\right)-c_{p}\left(T_{3 b}-T_{4}\right)}
$$

which by straightforward manipulation may be expressed in terms of dimensionless temperatures as:

$$
\frac{\tau-\theta}{(\tau-1)-\theta+(\theta-\epsilon\{\theta-1\}) / \tau_{e}} .
$$

(Note that the unrecuperated cycle expression may be recovered from eq.(18) by setting $\epsilon=0$.) 
Eq.(18) is plotted in fig. 9 as a function of $\tau$ and shows that (for the specified loss parameters) a recuperator effectiveness of at least $90 \%$ would be required for the recuperated cycle to offer any advantage over the simple cycle. Such a high effectiveness would be difficult to obtain in practice because the hot and cold streams in the heat exchanger have the same heat capacity. (Under these circumstances, an NTU of 9 would be needed (see, for example, [20]), implying a very large heat exchanger.) For lower compression and expansion efficiencies, the benefits of recuperation are more pronounced, but the performance is probably then too poor for the device to be worthwhile. (In this respect, Sisto [18] and Dieckmann et al [19] both reported improved performance with recuperation, but in cases where COPs were about 2 and 1.3 respectively.) Nonetheless, one obvious advantage of the recuperated cycle is that lower pressure ratios are required since part of the temperature rise $\left(T_{2}-T_{1}\right)$ occurs within the recuperator.

Recuperation also has a greater effect for lower radiator water return temperatures (i.e., lower $\theta$ ) and so might prove useful if the heat pump is integrated with an underfloor heating system. However, the improvements remain marginal for practical values of $\epsilon$ and may well be nullified by the additional pressure losses that would result from the larger heat exchange area and the increased flow complexity.

\section{CONCLUSIONS}

Cycle calculations have been presented for a recuperated and a simple cycle reverse Joule-Brayton heat pump at conditions appropriate for domestic cen- 
tral heating. Due to its inherently poor work ratio, the simple cycle suffers a dramatic deterioration in performance for small pressure losses and other irreversibilities. Recuperation renders the cycle less sensitive to such losses but, for realistic values of recuperator effectiveness, the improvement is only significant when losses are already high and the COP low.

As with all heat pumps, the performance depends on the temperature ratio over which the device operates and, in particular, the return water temperature from the heat distribution system (radiators, underfloor heating etc.) plays a dominant role in controlling the maximum COP that can be obtained.

In order to achieve a worthwhile performance, losses must be contained to within very low levels; typically compression and expansion efficiencies of about $99 \%$ are required, whilst overall pressure losses need to be less than $\sim 1 \%$. A preliminary consideration of the loss mechanisms suggests that these values may not be impossible, but further investigation is needed, particularly in connection with thermal dissipation losses. Heat losses and gains to and from the surroundings may also severely impair the COP, especially if the fractional heat gain in the expander falls below the fractional heat loss from the compressor.

\section{APPENDIX A: ESTIMATION OF PRESSURE LOSSES}

Accurate computation of pressure losses is a complex matter, requiring detailed information on the heat exchanger, valve and pipework geometries, together with consideration of Reynolds number effects and the pulsating nature 
of the flow. The intention here, however, is to provide approximate estimates based on dynamic head and frictional losses. The outline geometric details required to make these estimates are listed in table 1.

\section{Valve Losses}

Valve losses will be minimised if the valves open and close rapidly and are actuated when there is zero pressure difference across them. If this can be achieved then pressure losses will be limited to the loss in dynamic pressure of the valve flow, which may be estimated as:

$$
\Delta p=\frac{1}{2} \rho v_{p}^{2} / \sigma^{2}
$$

where $v_{p}$ is the maximum piston velocity and $\sigma$ is the ratio of valve freeflow area (including the effects of venae contractae) to piston area. Assuming sinusoidal piston motion,

$$
v_{p}=\frac{\pi \dot{m}}{\rho A_{p}}
$$

where $A_{p}$ is the piston cross-sectional area and $\dot{m}$ is the air mass flow rate. By locating valves in the cylinder end-walls and in the pistons, it is possible to obtain high values of $\sigma$, thereby keeping peak velocities to low levels. Using the figures listed in table 1, the following loss factors are obtained: 


\section{Compressor inlet valve: $0.79 \%$}

Compressor exit valve: $\quad 0.24 \%$

Expander inlet valve: $\quad 0.23 \%$

Expander exit valve: $\quad 0.77 \%$

\section{Heat Exchanger and Pipework Losses}

In order to estimate heat exchanger pressure losses, data for a compact heat exchanger of surface type 8.0-3/8T has been employed, as described in Kays and London [21]. Based on this data, an overall heat transfer coefficient of $U=50 \mathrm{~W} / \mathrm{m}^{2}$ has been assumed, with a corresponding friction factor of $f_{v}=$ 0.03. The required heat exchange surface is given by,

$$
A_{x}=\frac{\dot{Q}}{U \Delta T_{m}} \approx 20 \mathrm{~m}^{2}
$$

which can be achieved within a cubic volume of approximately $0.33 \mathrm{~m}$ side. The pressure drop associated with flow across the finned tube banks is:

$$
\Delta P=\frac{1}{2} \rho v_{x}^{2} f_{v}\left(A_{x} / A_{f f}\right),
$$

where $v_{x}$ is the maximum air velocity in the heat exchanger occurring at the minimum free-flow area, $A_{f f}$. Inserting values for the are ratios for surface 8.0-3/8T gives a fractional pressure loss of $\sim 0.1 \%$ at the reference operating conditions. A further pressure drop results from losses within the pipework connecting the compressor, heat exchanger and expander, but this cannot be computed without precise geometric details. It is assumed instead that this 
pressure drop is equal to the loss in dynamic pressure associated with the minimum flow area. Based on an estimated minimum pipework diameter of $0.1 \mathrm{~m}$, this gives a fractional pressure drop of a further $0.5 \%$.

For accurate calculations at conditions away from the reference operating point, values of $f$ should be recomputed taking account of changes in density, pressure and temperature. To a good approximation, however, the overall pressure loss factor may be written as:

$$
F \approx \sum f_{i} \propto \dot{m}^{2}
$$

where the summation is over all pressure loss factors. Using the above estimates, the overall pressure loss factor at the reference conditions is $F_{*} \approx 2.6 \%$. Since this figure is clearly subject to considerable uncertainty, the sensitivity of results to $F_{*}$ is considered in section 5.1.

\section{APPENDIX B: COMPRESSION AND EXPANSION EFFICIENCY}

Most applications of reciprocating compression and expansion devices relate to internal combustion engines operating at high speeds and high pressure ratios, or situations where the aim is to minimise compression work without regard for the final air temperature (and thus where cooled compression is beneficial). It is therefore difficult to obtain published data on compression and expansion efficiencies relevant to the current application. Moss et al [22] have estimated that (isentropic) efficiencies of $97.5 \%$ and $99.3 \%$ can be achieved for compression and expansion respectively for their design of a reciprocating Joule-Brayton CHP plant, but these are estimates rather than measurements 
and include a number of mechanical loss mechanisms not relevant in the current context.

The polytropic efficiencies used in the current work serve chiefly to represent thermal dissipation effects, since valve losses are included in the pressure losses discussed above. Thermal dissipation occurs even if the cylinders are externally insulated, since heat transfer will still take place to and from the cylinder walls. A number of studies have been undertaken to quantify this effect (e.g. [23,24]), particularly in connection with so-called gas springs. These comprise a quantity of gas trapped within a reciprocating piston and cylinder arrangement. The ideal gas spring would be fully reversible giving coincident compression and expansion lines on the $\mathrm{p}-\mathrm{V}$ diagram, but in reality a hysteresis loss results from thermal dissipation such that there is a net work input per cycle. An analytical expression for the hysteresis loss was developed by Lee [23] and has proved to fit experimental data well [24]. Lee's result is shown in fig. 10, expressed as an approximate decrement in polytropic efficiency by attributing half the loss to compression and half to expansion. The horizontal axis in this figure is the Peclet number, $\mathrm{Pe}=\omega D_{h}^{2} / 2 \alpha_{t}$, where $\omega$ is the angular frequency of the piston motion, $D_{h}$ is the cylinder mean hydraulic diameter, and $\alpha_{t}$ is the mean value of thermal diffusivity for air. Based on the dimensions and conditions listed in table 1 , the Peclet number is approximately 8000 , suggesting an efficiency loss of well below $0.5 \%$. However, there are two principal reasons why the thermal dissipation loss in a real compressor or expander (as opposed to a gas spring) may be substantially different:

(i) Flow through inlet valves will generate eddying motion and turbulence 
which will enhance the effective thermal diffusivity and thereby reduce the Peclet number.

(ii) The analysis leading to fig. 10 assumes a constant wall temperature. This is valid in the case of metal walls, but if the internal surfaces of the cylinder are constructed from insulating materials then the analysis should be extended to account for non-isothermal wall conditions.

Given these uncertainties, the calculations presented in sections 4.2 and 5.1 have been undertaken for a range of polytropic efficiencies, which are assumed equal for compression and expansion and independent of the mass flow rate.

\section{NOTATION}

A area, $\left[\mathrm{m}^{2}\right]$

COP coefficient of performance, $q / w$

$c_{p} \quad$ isobaric specific heat capacity, $[\mathrm{J} / \mathrm{kgK}]$

$D, D_{h} \quad$ diameter, hydraulic diameter $(4 \bar{V} / \bar{A}),[m]$

$f, F \quad$ pressure loss factors

$q \quad$ specific thermal output, $[\mathrm{J} / \mathrm{kg}]$

T temperature, $\mathrm{K}$

$w \quad$ specific work input, $[\mathrm{J} / \mathrm{kg}]$

$\alpha \quad$ heat loss / gain factor

$\alpha_{t} \quad$ thermal diffusivity for air, $\left[\mathrm{m}^{2} / \mathrm{s}\right]$

$\beta \quad$ pressure ratio

$\gamma \quad$ isentropic index

$\epsilon \quad$ heat exchanger effectiveness 
$\phi \quad$ polytropic index

$\eta \quad$ polytropic efficiency

$\rho \quad$ density, $\left[\mathrm{kg} / \mathrm{m}^{3}\right]$

$\tau \quad$ temperature ratio, $T_{2} / T_{1}$

$\tau_{c}, \tau_{e} \quad$ compressor, expander temperature ratio

$\tau_{c}, \tau_{e} \quad$ angular frequency of piston motion, $[\mathrm{rad} / \mathrm{s}]$

\section{subscripts}

$c, e \quad$ compressor, expander

$i \quad$ ideal cycle

1 heat pump intake

$2 \quad$ compressor delivery

$3 \quad$ main heat exchanger exit

$4 \quad$ heat pump exit

\section{References}

[1] J. Cockroft and N. Kelly, "A comparative assessment of future heat and power sources for the uk domestic sector," Energy Conversion and Management, vol. 47, pp. 2349-2360, 2006.

[2] D. Reay, Heat Pumps. Pergamon, 1988.

[3] J. Ewing, The mechanical production of cold. Cambridge University Press, first ed., 1908.

[4] G. Angelion and C. Invernezzi, "Prospects for real-gas reversed brayton cycle heat pumps," Int. J. Refrigeration, vol. 18, no. 4, pp. 272-280, 1995.

[5] J. Fleming, L. Li, and B. Van der Wekken, "Air cycle cooling and heating, part 2: 
A mathematical model for the transient behaviour of fixed matrix regenerators," Int. J. Energy Research, vol. 22, 1998.

[6] J. Braun, P. Bansal, and E. Groll, "Energy efficiency analysis of air cycle heat pump dryers," Int. J. Refrigeration, vol. 25, no. 7, pp. 954-965, 2002.

[7] C. Wu, L. Chen, and F. Sun, "Optimisation of steady flow refrigeration cycles," Int. J. Ambient Energy, vol. 17, pp. 199-206, 1996.

[8] C. Wu, L. Chen, and F. Sun, "Optimization of steady flow heat pumps," Energy Convers. Mgnt, vol. 39, no. 5, pp. 445-453, 1998.

[9] L. Chen, N. Ni, F. Sun, and C. Wu, "Performance of real regenerated air heat pumps," Int. J. Pow. Energy Sys., vol. 19, no. 3, pp. 231-238, 1999.

[10] L. Chen, N. Ni, C. Wu, and F. Sun, "Performance analysis of a closed regenerated heat pump with internal irreversibilities," Int. J. Energy Res., vol. 23, pp. 1039-1050, 1999.

[11] N. Ni, L. Chen, C. Wu, and F. Sun, "Performance analysis for endoreversible closed regenerated brayton heat pump cycles," Energy Convers. Mgnt., vol. 40, no. 4, pp. 393-406, 1999.

[12] L. Chen, S. Zhou, F. Sun, and C. Wu, "Performance of heat-transfer irreversible regenerated Brayton refrigerators," J. Phys. D: Appl. Phys., vol. 34, pp. 830$837,2001$.

[13] L. Chen, N. Ni, C. Wu, and F. Sun, "Heating load vs. cop characteristics for irreversible air-heat pump cycles," Int. J. Pow. Energy Sys., vol. 21, no. 2, pp. 105-111, 2001.

[14] Y. Zhang, J. Chen, J. He, and C. Wu, "Comparison on the optimum performances of the irreversible Brayton refrigeration with regeneration and non-regeneration," Applied Thermal Engineering, vol. 27, pp. 401-407, 2007.

[15] W. Swift, M. Nellis, G.F. amd Zagarola, J. McCormick, H. Sixsmith, and J. Gibbon, "Developments in turbo Brayton technology for low temperature applications," Cryogenics, vol. 39, pp. 989-995, 1999.

[16] J. He, Y. Xin, and X. He, "Performance optimization of quantum Brayton refrigeration cycle working with spin systems," Applied Energy, vol. 84, pp. 176$186,2007$.

[17] K. Sridhar, A. Nanjundan, M. Gottmann, T. Swanson, and J. Didion, "Evaluation of a reverse Brayton cycle heat pump for lunar base cooling," in 24th Int. Conf. on Environmental Systems, (Friedrichshafen, Germany), June 1994.

[18] F. Sisto, "The reversed Brayton cycle heat pump - a natural open cycle for HVAC applications," J. Eng. for Power, Trans. ASME, vol. 101, pp. 162-167, 1979. 
[19] J. Dieckmann, A. Erickson, A. Harvey, and W. Toscano, "Research and development of an air-cycle heat pump water heater." Final Report for Foster-Miller Associates, Inc., Waltham MA, 1979. Available at: http://adsabs.harvard.edu/abs/1979fmai.rept.....D.

[20] F. Incropera and D. Dewitt, Fundamentals of heat and mass transfer. Hobokon, 5th ed., 2002.

[21] W. Kays and A. London, Compact Heat Exchangers. New York: McGraw-Hill, fifth ed., 1984.

[22] R. Moss, A. Roskilly, and S. Nanda, "Reciprocating Joule-cycle engine for domestic chp systems," Applied Energy, vol. 80, pp. 169-185, 2005.

[23] K. Lee, "A simplistic model of cyclic heat transfer phenomena in closed spaces," in Proceedings of the 18th IECEC, pp. 720-723, 1983.

[24] A. Kornhauser and J. Smith, "The effect of heat transfer on gas spring performance," Transactions of the ASME, J. of Energy Resources Technology, vol. 115, pp. 70-75, 1993. 


\section{List of Figures}

1 Simplified layout of the reverse Joule-Brayton cycle heat pump.

2 Comparison of the real and ideal cycles on a T-s diagram. The two cycles are at the same operating point, as defined by the three temperatures, $T_{1}, T_{2}$ and $T_{3}$. Processes 1-1a, 2-2a etc. represent pressure losses in the real cycle.

3 The p-v digram for the ideal reverse Joule-Brayton cycle with a pressure ratio of 2:1 and a heat exchanger temperature drop of (a) $10{ }^{\circ} \mathrm{C}$ (solid line) and (b) $20{ }^{\circ} \mathrm{C}$ (broken line).

4 T-s diagrams showing the effects of heat loss and heat gain in the compressor and expander: (a) no heat losses, (b) $\alpha_{c}=\alpha_{e}=5 \%$ and (c) $\alpha_{c}=5 \%, \alpha_{e}=-5 \%$. Conditions correspond to the reference operating point defined in table 1 . All three cycles are reversible.

$5 \quad$ Variation of COP with overall pressure loss factor $F$ for different values of polytropic efficiency, $\eta$. The curves are plotted at the reference conditions, $\tau=1.216, \theta=1.18$.

6 Variation of COP with $\tau$ for different values of $\eta$, and $F_{*}$. Each set of curves is plotted for $F_{*}=1.25,2.5$ and $5 \%$. All curves are for $\theta=1.18$. 
7 Contours of COP plotted against $\tau$ and $\theta$ for $\eta=99 \%$ and $F_{*}=2.6 \%$. The open circle represents the optimum conditions for achieving a COP of 4.5.

8 T-s diagram for the recuperated cycle. Processes 1-1b and 3-3b represent heat exchange in the recuperator. Pressure losses have been omitted for clarity.

$9 \quad$ Variation of COP with $\tau$ for the recuperated cycle with different values of heat exchanger effectiveness. All curves are plotted for $\theta=1.18, \eta_{e}=\eta_{c}=99 \%$ and $F_{*}=2.6 \%$.

10 Loss in compression efficiency due to thermal dissipation in gas springs. Taken from the analysis by Lee [23] and plotted for a pressure ratio of 2.0 .

\section{List of Tables}

1 Reference operating conditions and outline geometry used to estimate pressure losses. 


\section{FIGURES}

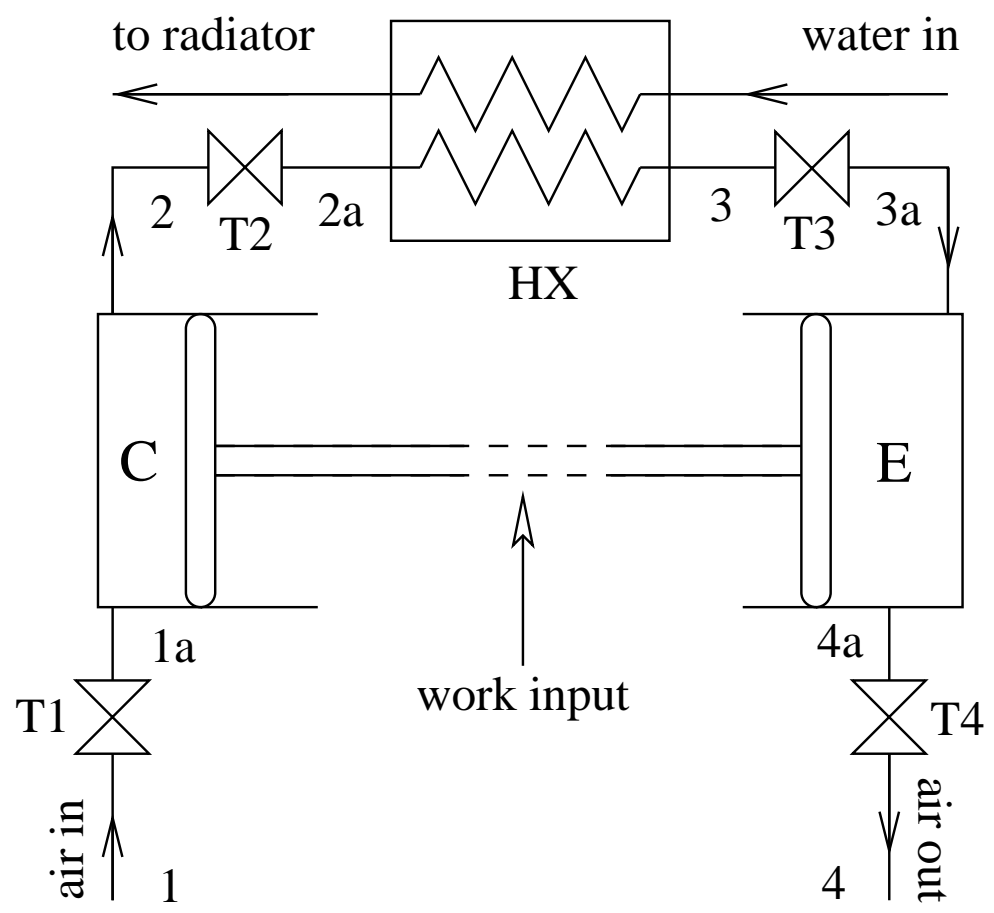

Figure 1. Simplified layout of the reverse Joule-Brayton cycle heat pump. 


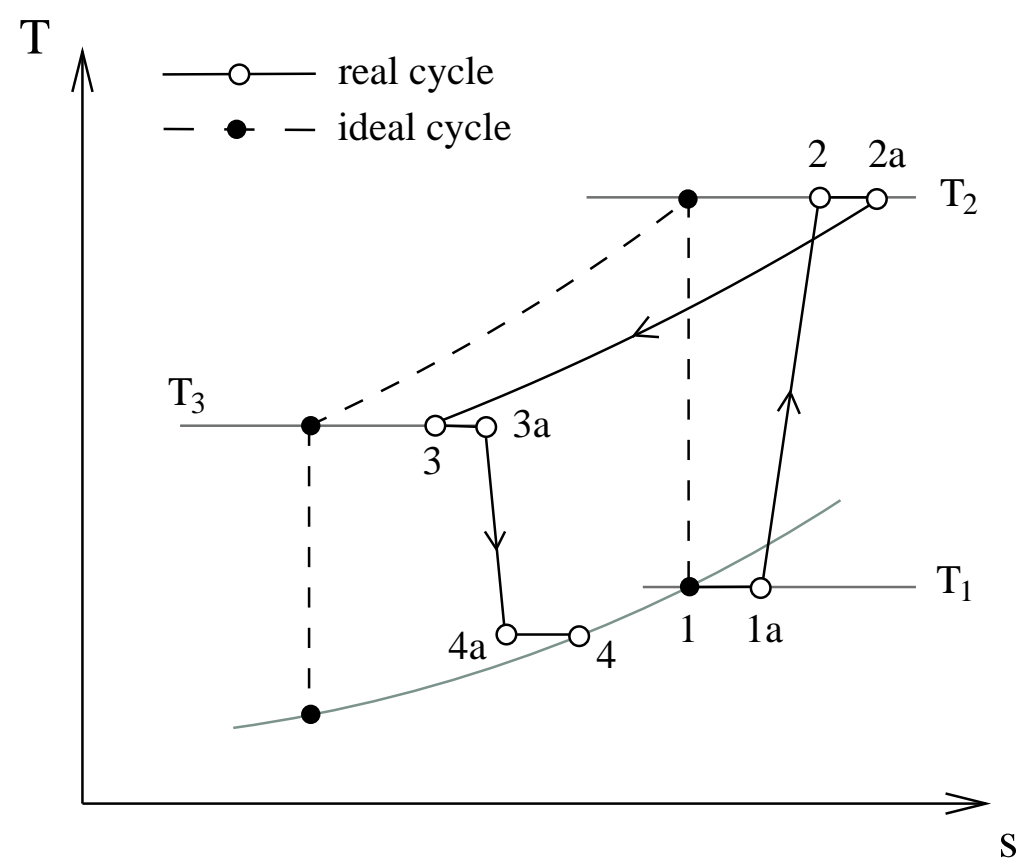

Figure 2. Comparison of the real and ideal cycles on a T-s diagram. The two cycles are at the same operating point, as defined by the three temperatures, $T_{1}, T_{2}$ and $T_{3}$. Processes 1-1a, 2-2a etc. represent pressure losses in the real cycle. 


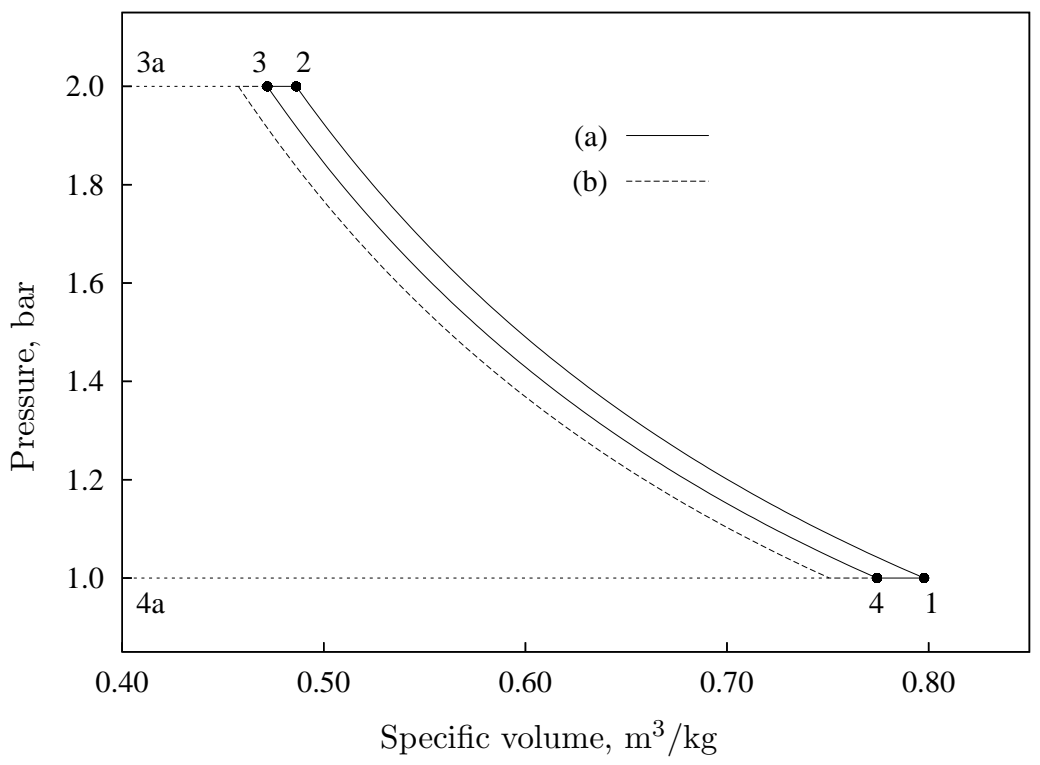

Figure 3. The p-v digram for the ideal reverse Joule-Brayton cycle with a pressure ratio of $2: 1$ and a heat exchanger temperature drop of (a) $10^{\circ} \mathrm{C}$ (solid line) and (b) $20{ }^{\circ} \mathrm{C}$ (broken line). 


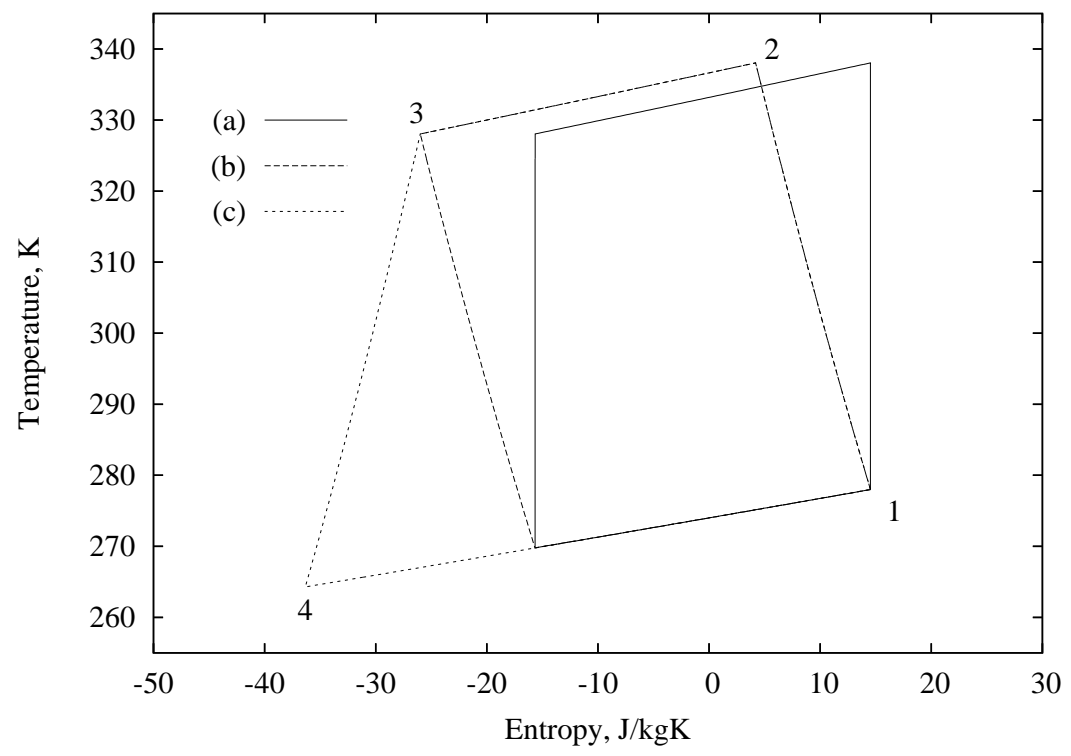

Figure 4. T-s diagrams showing the effects of heat loss and heat gain in the compressor and expander: (a) no heat losses, (b) $\alpha_{c}=\alpha_{e}=5 \%$ and (c) $\alpha_{c}=5 \%, \alpha_{e}=-5 \%$. Conditions correspond to the reference operating point defined in table 1 . All three cycles are reversible. 


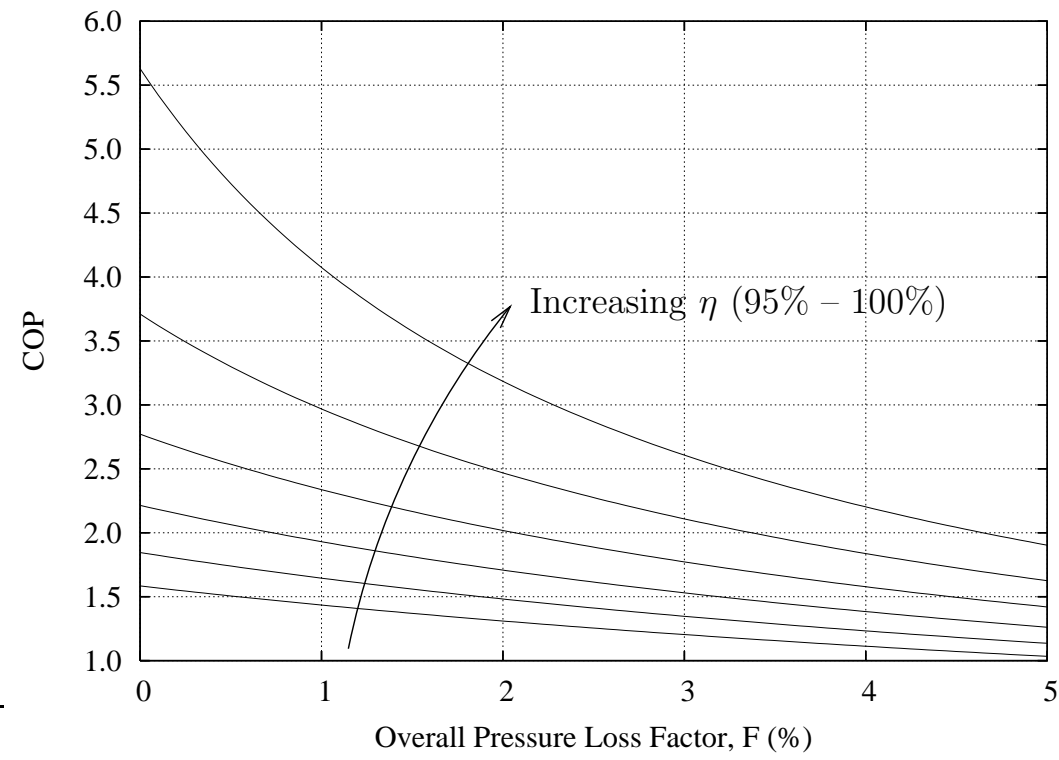

Figure 5. Variation of COP with overall pressure loss factor $F$ for different values of polytropic efficiency, $\eta$. The curves are plotted at the reference conditions, $\tau=1.216, \theta=1.18$. 


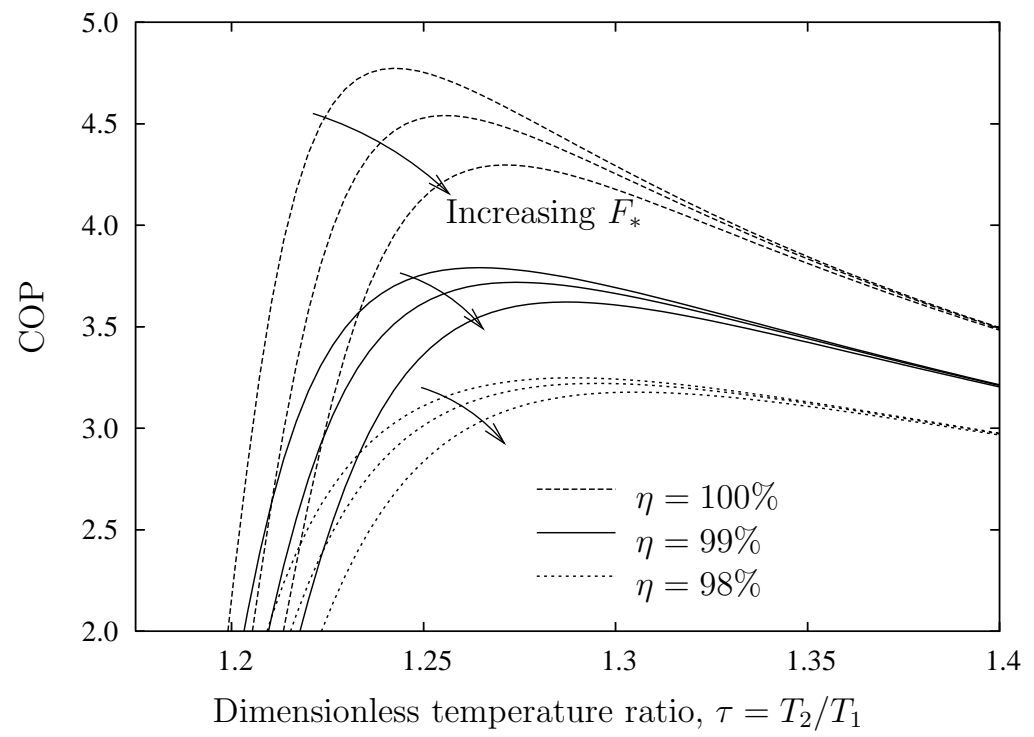

Figure 6. Variation of COP with $\tau$ for different values of $\eta$, and $F_{*}$. Each set of curves is plotted for $F_{*}=1.25,2.5$ and $5 \%$. All curves are for $\theta=1.18$. 


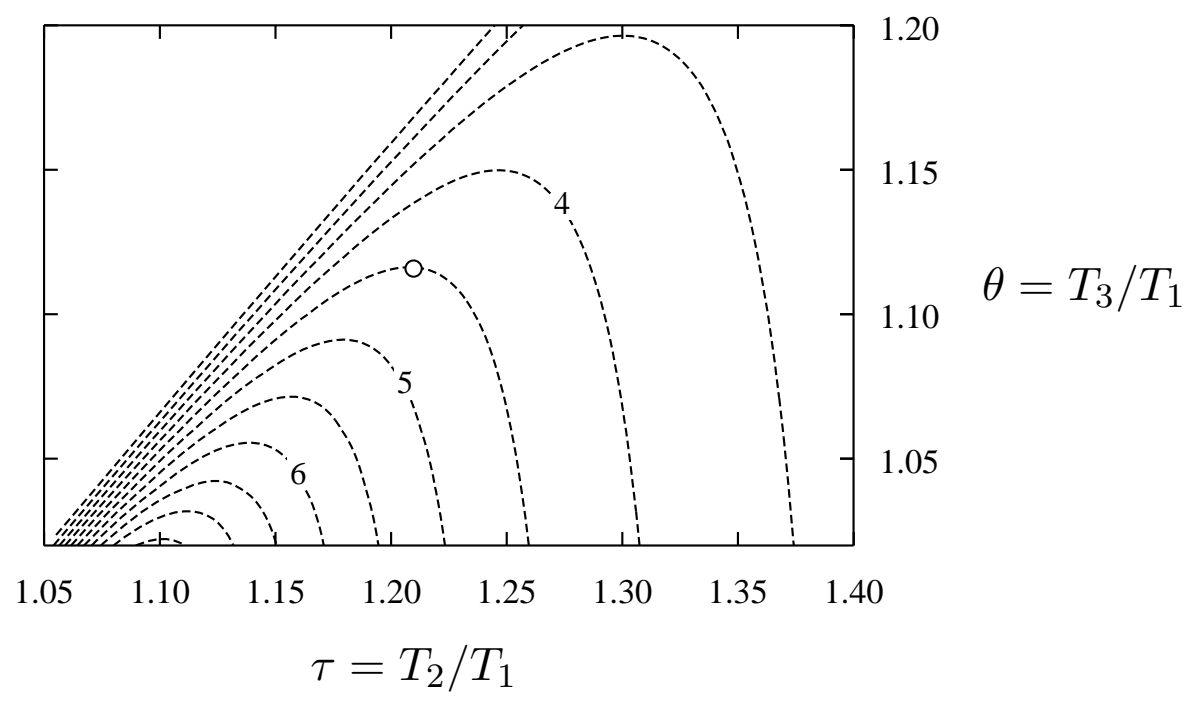

Figure 7. Contours of COP plotted against $\tau$ and $\theta$ for $\eta=99 \%$ and $F_{*}=2.6 \%$. The open circle represents the optimum conditions for achieving a COP of 4.5 . 


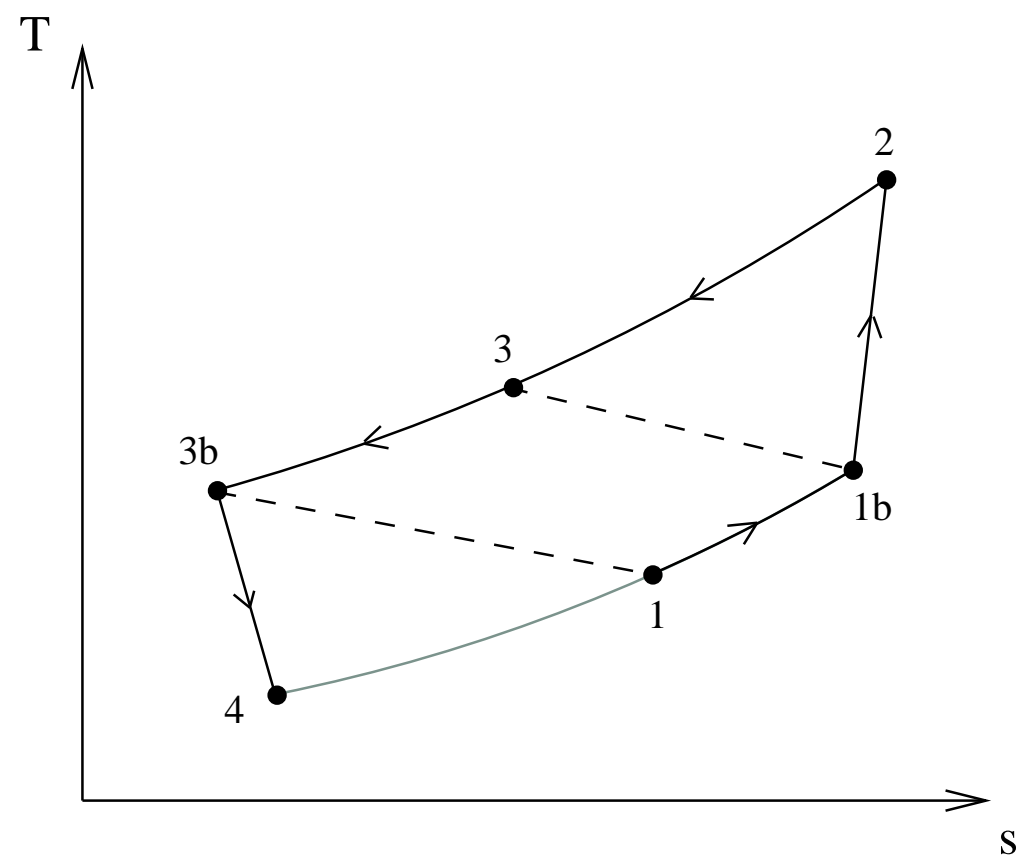

Figure 8. T-s diagram for the recuperated cycle. Processes 1-1b and 3-3b represent heat exchange in the recuperator. Pressure losses have been omitted for clarity. 


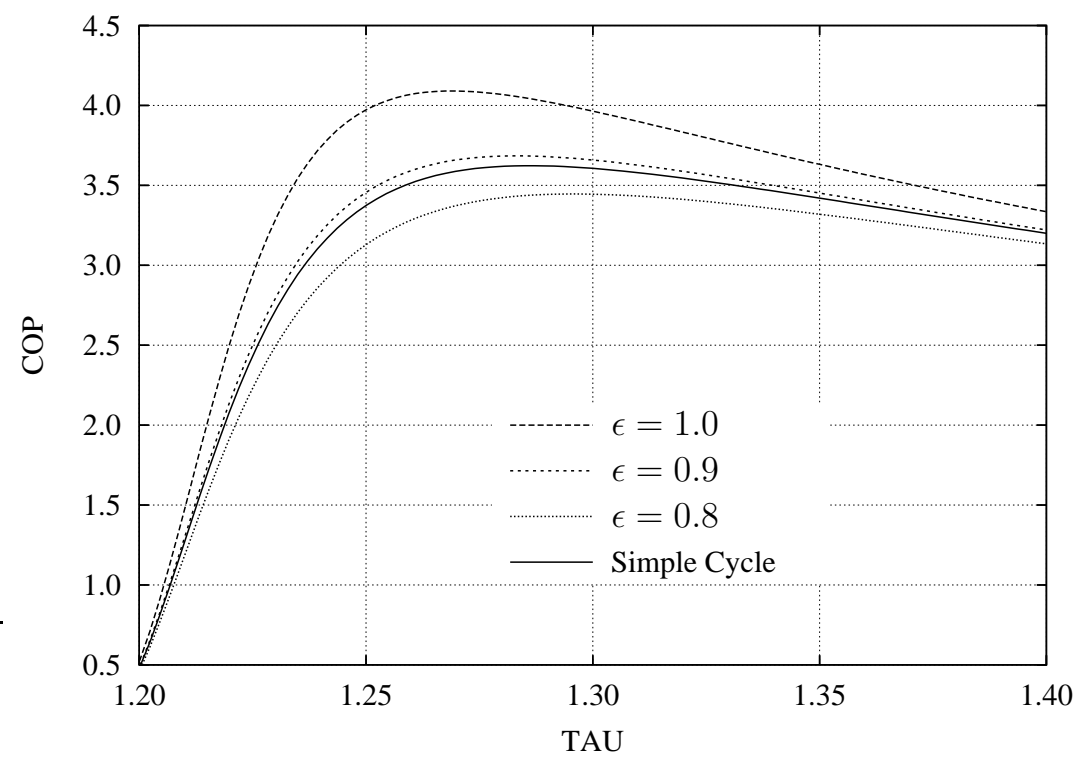

Figure 9. Variation of COP with $\tau$ for the recuperated cycle with different values of heat exchanger effectiveness. All curves are plotted for $\theta=1.18, \eta_{e}=\eta_{c}=99 \%$ and $F_{*}=2.6 \%$. 


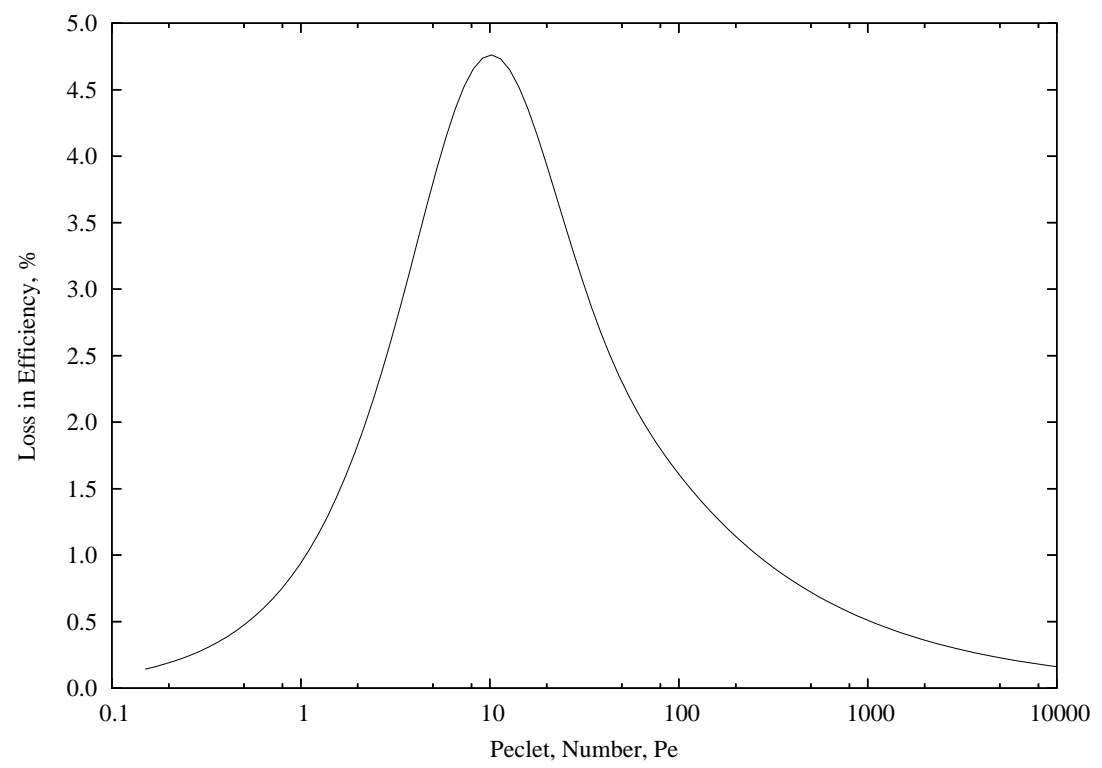

Figure 10. Loss in compression efficiency due to thermal dissipation in gas springs. Taken from the analysis by Lee [23] and plotted for a pressure ratio of 2.0. 
TABLES

Table 1

\begin{tabular}{|ll|c|}
\hline Number of compression cylinders & & 2 \\
\hline Piston diameter & $\sigma$ & $0.3 \mathrm{~m}$ \\
\hline Valve open area : piston area & $\dot{Q}$ & $5 \mathrm{~kW}$ \\
\hline Thermal output & $\dot{m}$ & $0.5 \mathrm{~kg} / \mathrm{s}$ \\
\hline Air-water log-mean temperature difference & $\Delta T_{m}$ & $5{ }^{\circ} \mathrm{C}$ \\
\hline Air mass flow rate & $T_{1}$ & $5{ }^{\circ} \mathrm{C}$ \\
\hline Ambient air temperature & $T_{2}$ & $65{ }^{\circ} \mathrm{C}$ \\
\hline Maximum cycle temperature & $T_{3}$ & $55^{\circ} \mathrm{C}$ \\
\hline Post heat-exchange air temperature & & \\
\hline
\end{tabular}

Reference operating conditions and outline geometry used to estimate pressure losses. 